\title{
Environmental sustainability and urban densification
}

\author{
S. Fatone, E. Conticelli \& S. Tondelli \\ Department of Architecture and Spatial Planning, \\ Alma Mater Studiorum, University of Bologna, Italy
}

\begin{abstract}
Densification is generally proposed as the solution to the excessive soil consumption that has characterized the urban development of European cities in the recent decades. Even if the "compact city" can generally be regarded as a preferred urban form over the "dispersed city", the strategy of densification is not applicable to any context, leaving aside the local conditions. Densification operations should consider the state of the environmental and urban context and assess threats and opportunities offered by the specific site. Thus, this paper describes a method for the environmental sustainability analysis of the built urban fabric. It proposes a set of sustainability objectives and pre-conditions to be evaluated in order to estimate the possibility to increase the urban density without compromising the environment, and it defines implementation measures, declined for the various environmental aspects which are considered to be affected by the densification interventions (water, soil, energy, electromagnetism, seismic, public facilities, waste, mobility, noise, air). The implementation measures are considered as guidelines for the inclusion of new volumes or for the completion of existing ones into the built areas, to be codified in the municipal building rules in order to guarantee the sustainability of the densification. The theme is explored both at the building scale and the urban scale, considering both the environmental aspects dealing with the building, such as the energy and seismic improvement, the urban morphology and the infrastructures capacity at urban scale, such as transport and green networks, water and road traffic flows.
\end{abstract}

Keywords: urban density, densification, environmental sustainability, compact city, building rules. 


\section{Introduction}

Sustainable development is widely recognized as a main goal for every government in the World (Jenks et al. [1]). Since nowadays more than a half of the world's population lives in town and cities, urban policies and plans can play a leading role in pursuing sustainability objectives, such as reduction of energy consumptions and of climate-changing emissions.

In the field of urban planning, the assumption of sustainability principles means the adoption of urban development models, which are aware of resources consumption and of the need to reduce environmental impacts. In industrialized countries, this gave mainly rise to developing processes referring to the so-called "compact city", considered as the opposite of urban sprawl (Neuman [2], Indovina [3]), the phenomenon which has characterized urban development in the last century and that is currently argued to be no longer sustainable. The high soil consumption, urban congestion, rising of infrastructure costs and people's worsening health are just some of the effects due to urban sprawl (EEA [4]).

The compact city model is generally considered able to remedy to this situation, by limiting the soil consumption and, therefore, by optimizing energy and transport flows, thanks to the enhancement of public transport, which can take advantage of the reduced distances among dwellings, work places and public facilities and, consequently, by reducing air pollutants and noise emissions (Neuman [2]).

Nevertheless, the paradigm of the compact city and of its connection to sustainability has been challenged with reference to the possibility of guaranteeing more liveable, efficient and attractive environments in respect of low density suburban areas (Breheny [5]), which can be endowed with higher urban and environmental quality. In fact, not always the relation between compactness and sustainability could be positively set, instead it could be weakly related, or correlated in limited ways (Neuman [2]). A high-density development could generate negative aspects on the built environment quality, such as higher traffic congestion and higher air and noise pollution levels, creating conflicts between land uses. This is one of the reasons why many European and North American areas have been affected by diffusion phenomena, characterised by the movement of the wealthier population towards suburban areas that guarantee higher building and life quality, leaving to the poorest population denser and less liveable areas, with an old and low quality building stock.

The adoption of the compact city model in the framework of sustainable development thus means the enhancement of densification policies, that are able to combine the quantitative concept of density with parameters and requirements guaranteeing high quality urban structures (Gibelli and Salzano [6]). In other words, density should be understood in terms of quality, by considering present and future scenarios with reference to environmental and social conditions of the site, in order to calibrate the interventions according to the context's spare capacity and the possibility to improve the quality of the urban context.

In this framework, densification policies should enhance sustainable development processes, aiming at enhancing the quality of the built environment 
by integrating new performances (i.e. energy, seismic, plants efficiency) and a proper land use mix, and, at the same time, at guaranteeing environment protection.

In fact, even if the "compact city" can generally be regarded as an urban form preferred over the "dispersed city" (Rogers [7]), densification is not a strategy applicable to any context, leaving aside the local conditions: in some cases, the carrying capacity of natural and anthropic components does not allow any further addition. Densification operations must therefore take into account the limitations and the opportunities offered by the site.

This paper describes a method for the environmental sustainability analysis of the built urban fabric. It proposes a set of sustainability objectives and preconditions to be evaluated in order to estimate the possibility to increase the urban density without compromising the environment, and defines implementation measures, declined for the various environmental aspects (water, soil, energy, electromagnetism, seismic, public facilities, waste, mobility, noise, air). The implementation measures are considered as guidelines for the inclusion of new building volumes or for completion of existing ones into the built areas, to be codified in the municipal building rules in order to guarantee the sustainability of the densification. Through the application to a case study - the densification of blocks of consolidated urban fabric in the Municipality of Faenza, in the Emilia-Romagna Region - the theme is explored both at the building and at the urban scale.

\section{The environmental sustainability assessment of densification policies}

The implementation of densification strategies means the assumption of a generally accepted sustainability objective: the reduction of soil consumption, aiming at conserving rural areas and natural resources. Nevertheless, this could reveal to be inadequate to guarantee urban sustainability because, while operating on the built environment, it is necessary, first of all, to avoid every modification of the existing environmental balance and, secondly, to remedy to eventually present critical situations, such as lacks in public facilities or infrastructures.

Thus, to determine the real potential of urban compactness, it is necessary to consider the changes of baseline levels of compactness that occur through the process of compaction (Burton [8]).

It is therefore necessary to assess the environmental sustainability of every densification policy, taking into account the impacts on every environmental aspect and the possible effects at local and municipal scale deriving from the increase in urban intensity on technological and road networks and on public facilities.

The sustainability at municipal scale is assessed through the Strategic Environmental Assessment, to be applied at the municipal structure plan (Piano Strutturale Comunale, PSC), which fixes limits and conditions to the proposed urban changes. Nevertheless, the PSC is a strategic plan and does not define the 
exact location of the new developments or of the volumes that can be employed to foster densification and renewal policies in the well-established urban fabric. According to the Emilia-Romagna town planning law n. 20/2000, two further planning tools complete the PSC: the municipal operative plan (Piano Operativo Comunale, POC), which assigns building capacity to the areas subjected to new developments and urban renewal, and the building and urban regulation (Regolamento Urbansitico ed Edilizio, RUE), which assigns the volumes to the specific areas within the built urban fabric, in respect of the limits and conditions defined by the PSC.

The paper describes a methodology for the environmental assessment of densification policies proposed by the RUE, hence acting within a framework of sustainability conditions assessed at municipal level by the PSC.

For each environmental aspect and for a given area (normally, corresponding to a block), defines:

- $\quad$ sustainability objectives that should be respected by the densification policy in order to guarantee the city sustainability and that should bring to modify the adopted policy if it appeared not coherent with sustainability objectives.

- pre-conditions, i.e. characteristics that the area or the entire urban system should have in order to allow the implementation of the densification; in other words, pre-conditions define the site suitability to densification and could identify factors excluding the interventions.

- implementation measures, that consist of specific indications and prescriptions aiming at guaranteeing the sustainability of the planned intervention.

- references (plans, laws, documents, etc.) connected to the proposed policy, whose respect guarantees the interventions coherence and the feasibility.

Through these steps, it is possible to define the "limiting factor" of the admitted densification in a specific area for each environmental aspect, i.e. the residual capacity of the more critical environmental aspect, which determines the maximum added urban development that can be considered sustainable for that area.

The environmental aspects that have been considered to be affected by densification policies are water (supply and disposal), soil (permeability, urban drainage), seismic grade, energy (supply, consumption), electromagnetism, waste, mobility, public facilities (parks, parking lots, schools, public interest services), air quality, noise. Some of them require a local assessment, at area or block level (e.g. permeability), or at building scale (e.g. energy), others refer to municipal scale balances (e.g. mobility, water, etc.).

The feasibility and usefulness of the proposed method have been verified through the application to a case study, the assessment of densification strategies proposed by Faenza's PSC). Faenza Local Administration boasts a long-time attention towards sustainability themes and it has introduced sustainability objectives inside the PSC, aiming at reducing soil consumption and at 
concentrating the whole urban growth of the next 15 years inside the build fabric, through enhancing densification operations.

The outcomes of the study are prescriptions and recommendations to be included into the Building and urban regulations (RUE) which are now under drafting.

\section{The case study: densification operations in Faenza}

The assessment of the environmental aspects has been carried on three standard situations which have been selected inside the town well-established urban fabric, which has been indicated by the PSC as the area where to carry on densification policies, excluding the buildings with historical or architectural value that could be compromised by the intervention, and aiming at increasing the compactness of the town by acting on the city edges.

First of all, in order to choose the intervention areas, the compactness of Faenza urban fabric has been measured through the Compactness Index CI and the shape coefficient SC (Salvetti [9]). CI indicates the extent to which an urban fabric is similar to the circle, which is considered to be the optimal surface, with $\mathrm{CI}=1$ and $\mathrm{SC}=\mathrm{SP} / \mathrm{CP}$ is the result of the ratio between the settlement perimeter (SP) and the perimeter of the circle (CP) having the same surface of the considered settlement. Faenza results a city of medium compactness $(\mathrm{CI}=0.5)$, but its shape coefficient is much higher that 1 because the perimeter of the settlement is very ragged, indicating that the intervention on the city edges is a priority.

Second, the analysis of the urban fabric allowed to select three sample areas belonging to the 1950s (blocks), 1970s (dwellings) and 1990s (mall) suburbs (fig. 1), which are characterised by the presence of depleted buildings, with very low energy and seismic performances. The samples area represents frequent buildings types in the consolidated urban fabric of the town, and they are suitable for densification intervention, thanks to very low Floor Area Ratios (FAR,

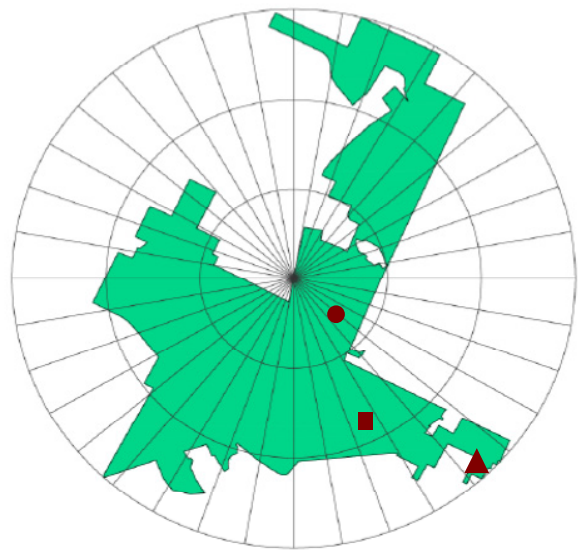

- One/two floors isolated dwellings

- High isolated blocks

$\Delta$ Shopping mall

Figure 1: $\quad$ Faenza's compactness index and location of the sample areas. 
expressed in the size of the footprint - square width - of the building and the height of the building, as compared with the overall square footage of the property lot). The three areas differ for land use and for the way densification can be undergone on them: the first is characterised by the presence of one or two floors isolated dwellings, the second has high isolated blocks and the third has a big and quite abandoned shopping mall with its parking lot.

Case study 1 - one floor dwelling in San Rocco neighbourhood

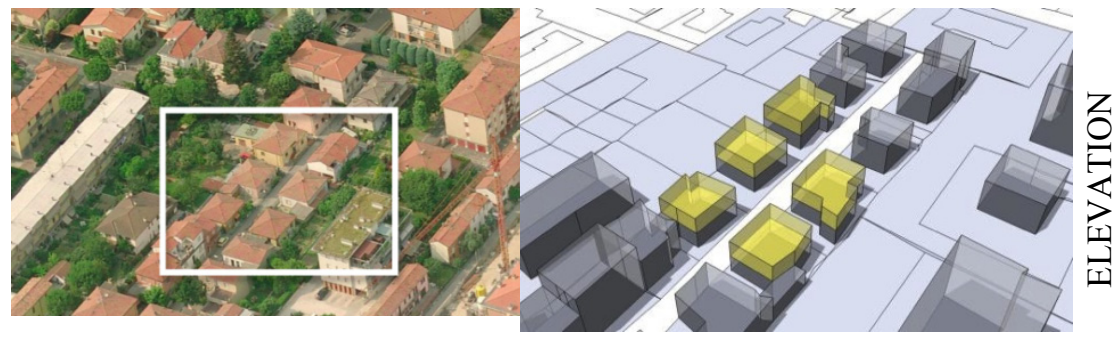

Case study 2 - high isolated blocks in Borgo neighbourhood
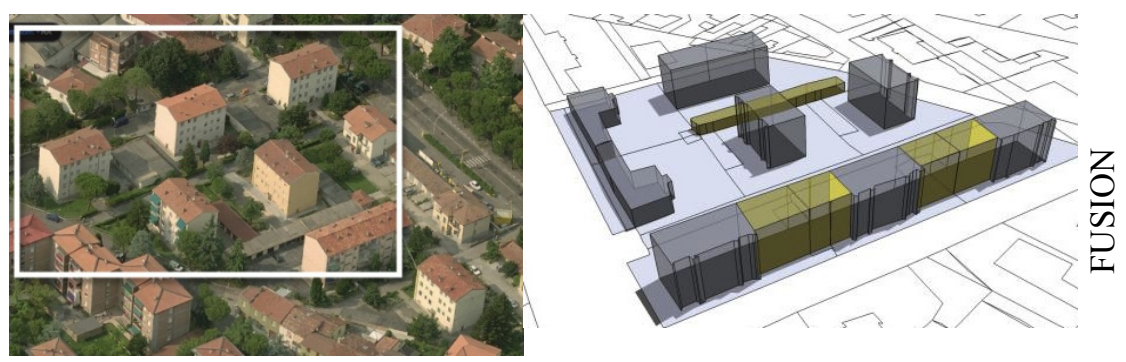

Case study 3 - shopping mall in Borgo neighbourhood

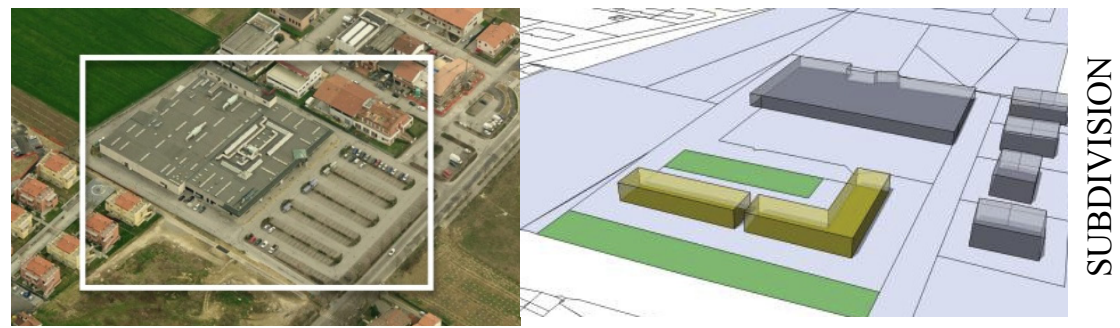

Figure 2: Aerial photo of the three sample areas and 3D models of the densification interventions.

For each environmental aspect, sustainability objectives have been selected according to the PSC strategies; then, preconditions and implementation measures have been defined and possible references have been indicated. When 
numerical data were available, a quantitative assessment of the expected environmental effects has been carried on; aspects, for which numerical data were not available, have been assessed on qualitative level. Furthermore, the environmental assessment has permitted to select the suitable densification strategy to be adopted in the different situations among many means or ways of achieving densification. i.e. construction of attached/detached second dwellings, extension of the building or adding on of floors, blocks elevation, blocks fusion, subdivision of land and infill with new blocks, demolition and integration of existing structures, infill on vacant and under-utilised land, etc. (Spatial Planning and Urban Design Department, City of Cape Town [10]).

To better describe the proposed methodology, the assessment for the first sample case is described below. The considered area is located in the north-east suburbs of Faenza, within San Rocco neighbourhood, and sees mainly the presence of single one or two floors dwellings. The block surface is about $2,000 \mathrm{~m}^{2}$, with a very low FAR (equal to 0.21 ). The densification proposal sets out the doubling of the built surface (about $400 \mathrm{~m}^{2}$ ), leading to a FAR of 0.42 (fig. 2).

\subsection{Soil}

Objective: permeability and water-bearing stratum recharge maintaining

Pre-conditions: the densification intervention should guarantee suitable soil permeability with reference to its classification. In the considered case, since the area is situated upon average permeable rocks and ground $\left(10^{-4}<\mathrm{K}<10^{-7} \mathrm{~m} / \mathrm{s}\right)$ within an underground waters protection zone, the Permeability Index (PI = covered surface/property lot) should not be lower than the present one, equal to 0.79 . This precondition appears to affect the possible strategy of densification that can be applied in this case, because only elevation intervention are admitted, fig.2, while others form of intervention outside the covered area of the present buildings would decrease PI.

Measures: natural or recovered draining ground surfaces should be adopted to allow a permeability increase.

References: PSC, Waters Protection Plan (PTA), sector laws

\subsection{Water}

Objective: waters sustainable management, thus guaranteeing the drink water supply, the sewage water disposal and hydraulic invariance

Pre-conditions: the residual capacity of the drink water network and of the purifier have already been assessed in the PSC strategic environmental assessment, by means of the analysis of the present network an facilities capacity, the projections of the water consumption according to the PSC targets (130 1/inhab/day) and the futures disposal needs (245 1/inhab/day). Because the purifier is unique for the whole city of Faenza, its capacity has been assessed considering the sum of all the densification interventions: since it can serve up to 100,000 equivalent inhabitants, and the present population of Faenza consists of 57,000 inhabitants, it can bear an even intensive densification action on the 
territory. Since the inhabitants' increase for the considered area, following the densification intervention, is contained within the PSC total volumes increase, the water networks and facilities can bear the estimated increase at local level and they do not make up a limit to the considered intervention.

Measures: to guarantee the intervention sustainability, it is necessary to set up separate sewage for allowing the reuse of rain and grey waters and to adopt devices for the consumptions reduction.

References: Waters Protection Plan (PTA), sector laws

\subsection{Energy}

Objective: increasing the renewable energy use and reducing consumption

Pre-conditions: at the urban scale, there are no pre-conditions that could forbid the densification intervention; the only condition to be fulfilled is the maintaining/reducing of the energy consumption from non-renewable sources, which requires the reduction of the existing buildings consumptions.

Measures: a deep analysis of the site-specific microclimate conditions is required in order to profit of sun light contribution, of natural ventilation, etc. At the building scale, the new buildings should guarantee high energy performances (energy class A), while it is necessary to improve the energy performances of the existing ones aiming at keeping the unit consumption $\left(\mathrm{kWh} / \mathrm{m}^{2}\right)$ of the total of the new constructions and the regeneration of the existing one, lower (or equal) to the previous one.

References: Energy Plan, sector laws

\subsection{Electromagnetism}

Objective: avoiding the exposure of the inhabitants to electromagnetic pollution sources

Pre-conditions: the absence of electromagnetic sources within the law thresholds already guarantees the intervention feasibility. In the considered case, a $132 \mathrm{kV}$ aerial line is present near the area; considering the normative thresholds and the distances between the lines and the buildings, the intervention is admitted.

Measures: densification interventions should minimize the impact of the various polluting sources that can be found in the urban context (transmitting antennas, TV aerials, etc.) by studying the buildings height in relation to the sources location.

References: PSC, Provincial Plan for radio and TV broadcasting (PLERT), sector laws

\subsection{Seismic}

Objective: guaranteeing the seismic feasibility aiming at the buildings static and dynamic safety in case of earthquake

Pre-conditions: at the urban scale, the municipal territory is subdivided into seismic micro zones indicating the local seismic danger with reference to 
buildings' geometric and typological characteristics, and to the interactions between buildings. At the building scale, the condition to be verified is its bearing capacity. In the case study, the area belongs to micro zone n.6, according to which an amplification factor equal to 1.7 has to be considered. The densification intervention is therefore admitted on condition that the seismic answer of the existing buildings is not made worse (fig. 3).
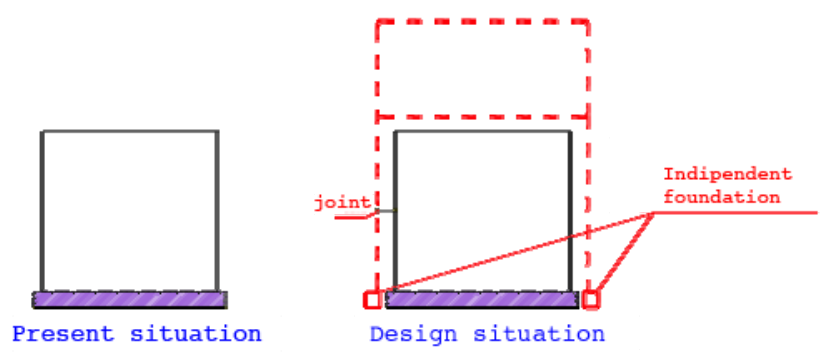

Figure 3: Scheme of a possible elevation intervention not making worse the seismic answer of the existing building.

Measures: a technical report is necessary, describing the employed materials and techniques and certifying the building seismic suitability.

References: sector laws

\subsection{Public facilities}

Objective: guaranteeing the public facilities quality and quantity

Pre-conditions: in the Emilia-Romagna Region, the minimum standard for public facilities (including parks, parking lots, schools, public facilities) in a town with more that 10,000 inhabitants is $30 \mathrm{~m}^{2} /$ inhab. The considered area belongs to the north-east part of the town, which has a very high standard ( $42 \mathrm{~m}^{2} /$ inhab); at municipal scale, the standard remains above the minimum $\left(34.7 \mathrm{~m}^{2} /\right.$ ihnab). Therefore the intervention can be admitted because the existing public equipment is able to serve the new inhabitants.

Measures: the densification intervention should guarantee the access to the public facilities by designing cycle and pedestrian paths and by connecting green areas.

References: PSC

\subsection{Waste}

Objective: guaranteeing separated waste collection

Pre-conditions: the increase in inhabitants' number generates an increase in the waste amount to be managed at municipal scale; therefore a pre-condition to the densification intervention is the verification of the disposal capacity of the existing facilities. In the case of Faenza, the community's waste depot has a 
residual capacity of the $30 \%$, therefore the densification interventions can be admitted.

Measures: aiming at enhancing the separated collection, suitable spaces for the containers should be provided in the property lot or inside the building.

References: sector laws

\subsection{Mobility}

Objective: maintaining or improving the mobility efficiency

Pre-conditions: the choice of the intervention area should take into account the presence of possible critical situations for what concerns mobility, considering the increase in origin/destination trips caused by the development and assessing the road network and public transport system capacity to face it. In case study n.1, the road network can easily face the trips increase and no congestion is foreseen.

Measures: the densification intervention should guarantee the connection to cycle and pedestrian paths.

References: Traffic Local Plan (PUT)

\subsection{Noise}

Objective: maintaining the acoustic climate

Pre-conditions: the densification intervention should be assessed according to the acoustic zoning, depending on the land use. The considered area is located into the III class zone, and is therefore suitable for residential uses.

Measures: The use of acoustic barriers in the new developments should be avoided for its negative impact on urban quality; therefore, the new buildings/parts of buildings should be designed in a protected location, far from noise sources, and the architectural design should take into account noise protection, by means of indentations, loggias, overhanging screens, etc.

References: acoustic zoning, PSC

\subsection{Air}

Objective: containing air pollutants emissions

Pre-conditions: the absence of polluting sources within the law thresholds already guarantees the intervention feasibility.

Measures: the changes in the spatial configuration of the blocks can affect the breezes speed and direction, while the increase in the inhabitants' number (and therefore in the generated and attracted trips) could increase air pollutants emissions. The densification intervention should guarantee the connection to cycle and pedestrian paths and with public transport systems and the blocks design should take into account the effects on microclimate.

References: Air quality provincial plan (PGQA), PUT, PSC 


\section{Discussion}

Starting from the notion of "compact city", the proposed assessing methodology has defined a set of rules aimed at boosting and leading densification interventions inside the existing urban fabric while ensuring high levels of quality and liveability.

The embedment of sustainability objectives is essential for managing every changes of the contemporary city, therefore the proposed method is based upon the integration of urban sustainability targets into the densification policies. The result is the definition of criteria and performances to be acknowledged in the planning tools and regulations, which will indicate the suitable interventions and the maximum densification level that will ensure the sustainability of the interventions.

The implementation of densification policies, with respect to the development of rural land or even the redevelopment of wide brownfields inside the city, represents a greater challenge, because it's based on more complex operations; nevertheless, urban densification may achieve higher advantages in improving the city environment, in particular in a phase like the present one, when Italian property market is undergoing a strong contraction and it suffers from an excessive building supply compared to a low population increase. Furthermore, Italy has a huge old building stock, which is responsible for the main inefficiencies and the low quality of the urban context, and no sustainability at city level can be achieved without the regeneration of the existing building stock.

Considering densification policies as volumetric incentives to be exploited to finance retrofitting or substitution interventions of the built real estate, as it's happening in Italy thanks to the Law n. 106/2011 already acknowledged in many regional laws, is an unmissable chance to lead a radical action on the performances of the built urban fabric, but this action needs to be assessed in order to guarantee its environmental sustainability. Otherwise, these interventions risk to worsen the present situation and the results of densification operations could be an unsustainable increase of the anthropic pressure on the environmental and urban context.

Urban and environmental complexity generates concurrent effects, which are often hard to foresee and which need to be assessed both at the municipal and at the local scale. In fact, on one hand, local interventions, that could appear as irrelevant if considered one by one, could interact to each other to produce a joint effect that is greater than the sum of the single estimated impacts. Therefore the assessment at local scale needs to stand by the environmental assessment of the urban policies at the municipal scale. On the other hand, the assessment at municipal scale risks to mediate the results and to forget some local limits to the transformation. In this framework, our paper suggests a methodology able to support the assessment of the sustainability of each densification intervention, but in the meantime able to consider the changes inside the limits fixed by the sustainability assessment of the development policies at municipal scale.

Secondly, the proposed methodology may be considered as an extremely flexible and simple operative logic, which can be applied to different situations 
or spatial and normative contexts and whom every Municipality, even the smaller ones that generally have a lower analytic capacity, can apply.

Including these logics inside the towns' building and urban regulations will permit to define the "spare building capacity" for every urban portion and, consequently, will permit to manage the even more necessary interventions in the well-consolidated urban fabric in the respect of sustainability principles.

\section{References}

[1] Jenks, M., Burton, E., and Williams, K., The Compact cities and sustainability: an introduction, Jenks, M., Burton, E., and Williams, K. (eds), The Compact city: a sustainable urban form?, E\&FN Spon: Oxford, 1996.

[2] Neuman, M., The Compact City Fallacy. In: Journal of Planning Education and Research, September, vol. 25, n. 1, pp 11-26, 2005.

[3] Indovina, F., Dalla città diffusa all'arcipelago metropolitano, Franco Angeli: Milano, 2009.

[4] European Environment Agency (EEA), Urban sprawl in Europe. The ignored challenge, Report n. 10, Office for Official Publications of the European Communities: Luxembourg, 2006.

[5] Breheny, M. Urban compaction: feasible and acceptable?, Cities n.14, pp. 209-217, 1997.

[6] Gibelli M.C., Salzano E. (eds), No sprawl, Alinea: Firenze, 2006.

[7] Rogers, R. (ed.), Towards a Strong Urban Renaissance, Urban Task Force: London, 2005.

[8] Burton, E., Measuring urban compactness in UK towns and cities, Environmental and planning B, Planning and Design, 29: 219 -250, 2002.

[9] Salvetti, A., I gradi di dispersione della popolazione e di compattezza degli insediamenti, in: Astengo, G. (ed.), Rapporto sullo stato dell'Urbanistica in Italia e sulle politiche urbanistiche e territoriali per gli anni '80, Ricerche MPI-IUAV, Venezia, 1982.

[10] The Spatial Planning and Urban Design Department, City of Cape Town, Cape Town Densification Strategy Technical Report, 2009. 